\title{
Débattre de l'intégration en France et en Belgique
}

Le débat sur l'intégration, en France comme en Belgique, s'inscrit trop souvent en creux.

Au lieu d'évoquer "ce à quoi nous voulons collectivement intégrer les étrangers",

il s'agit le plus souvent d'agiter stérilement arguments pour et contre, dans un face à face agressif entre ceux qui croient que lintégration est en marche et ceux qui pensent le contraire.

Qui, dans ces débats, parle d'une véritable proposition sociale?

Les pires débats sont ceux qui portent sur des objets indéfinis. Ils favorisent l'incompréhension, les frustrations, le désaccord, et n'apportent que très rarement des réponses appropriées. Leur spécificité réside dans le flou de leur contenu. Ces débats sont interminables, ils prennent souvent un ton agressif, et les gens finissent par s'en prendre personnellement les uns aux autres au lieu de démonter leurs idées respectives. Il ne peut en être autrement car personne ne sait mettre la main sur le désaccord premier.

Le "débat" public sur l'intégration en France et en Belgique francophone affiche bien ce processus. La plupart des acteurs engagés dans celui-ci ne peuvent se mettre d'accord, échanger des points de vue, s'enrichir mutuellement et établir un consensus dans ce domaine car ils ignorent la réalité, le fond, les enjeux dont ils se veulent pourtant les porte-paroles. Plus précisément, ils "sentent" ces derniers, ils les devinent mais n'arrivent pas à les dire, ni à les qualifier. Ils les "vivent" affectivement sans les identifier correctement, laissant la haine et les attaques peu convenables prendre le contrôle d'un débat impossible, insensé, inouï et finalement extravagant lorsque l'on regarde cela avec un peu de recul.

Le président du Mouvement réformateur belge (le MR, la droite francophone), Daniel Ducarme, ne pouvait mieux illustrer cela lorsque, à la mioctobre 2002, dans un élan qu'il considérait comme courageux à l'approche des élections fédérales de mai 2003, il annonçait que "la politique d'intégration des étrangers est un échec, que les personnes d'origine étrangère ne se sentent pas bien en Belgique et que les Belges de souche ne se sentent pas bien chez eux"(1). Un peu à la manière de ce qui se fait en France lorsqu'un élu reprend à son compte un thème appartenant au fond de commerce du Front national, un "débat" virulent et acerbe a vu rapidement le jour dans les tribunes de la presse et de la télévision pour finalement aboutir à un règlement de compte dominical, en direct, sur la principale chaîne publique francophone. Ce duel opposait celui qui avait osé ressortir les vieux démons "de la réussite ou de l'échec de l'intégration", et qui à ce titre vantait le courage d'une telle démarche, à ceux, en face, qui n'avaient pas l'intention de laisser une affirmation aussi simpliste ("l'intégration est par Jérôme Jamin, chercheur au Centre d'études de l'ethnicité et des migrations

(Cedem - université de Liège) et à l'Ethnicity and social policy research unit (ESPR university of Bradford)

1)- Lire, entre autres articles sur les propos du président du MR, La Libre Belgique et Le Soir du 16 octobre 2002. 
un échec") empoisonner tout ou partie de la future campagne électorale. Ce duel n'a finalement rien apporté de neuf dans ce domaine, pas plus que les nombreux billets publiés à cette époque dans la presse. Il en a été ainsi car, par une sorte d'aveuglement collectif, personne n'a véritablement cherché à mettre le doigt sur la réalité, le fond, les enjeux dont, de bonne foi, ils se voulaient tous pourtant les porte-paroles. Consciemment ou non, égaré, ébloui par la phrase expéditive du président du MR, emprisonné dans les méandres d'un débat mal fichu, sur un objet historiquement mal défini, chacun s'est contenté de batailler immédiatement, et une fois de plus, sur les preuves de la réussite ou de l'échec de l'intégration, et sur les conditions de possibilité d'une évaluation à son endroit.

Ce triste phénomène qui plombe et pervertit le débat public provient d'un réflexe tenace de "déplacement", dans l'ordre, du discours sur l'intégration, et de ses enjeux les plus fondamentaux. Cela consiste à passer de la question centrale - "ce à quoi nous voulons collectivement nous intégrer" -, à la question secondaire de "la réussite ou de l'échec de l'intégration". Ce faisant, il esquive le débat sur le sens et le contenu que nous donnons à la société (intégrée), et transpose d'autorité la réflexion sur le chaos ou le triomphe de l'intégration selon l'avis des uns ou des autres. Le déplacement transforme ainsi la recherche sur nous-mêmes, et le débat sur ce que nous voulons être et ce que nous sommes, en pourparlers dérisoires sur la prétendue faillite de l'intégration. Nous ne pourrons toutefois jamais évaluer celle-ci et en débattre intelligemment sans même avoir essayé d'établir, au préalable, un accord sur la définition de la société intégrée, sans avoir interrogé radicalement notre identité et ses marqueurs d'appartenance. La prise de conscience de ce déplacement ouvre les portes d'une solution à l'amiable et d'un consensus élaboré collectivement.

\section{Faux débats et vrais "déplacements"}

S'il abuse, trompe et mystifie ostensiblement, le "déplacement" fait toutefois partie intégrante de l'histoire de la phraséologie sur l'immigration et l'intégration depuis la Seconde Guerre mondiale. Situé au milieu d'une échelle à trois niveaux, entre la démarche de ceux qui veulent d'abord définir la société intégrée avant d'évaluer l'intégration, et la conduite de ceux qui ne veulent pas en entendre parler, persuadés que pour des raisons culturelles et biologiques l'intégration est impossible, la logique du déplacement anime avec une grande efficacité de nombreuses controverses sur la question. On la retrouve également, sans surprise, dans les principaux textes touchant à la naturalisation et à l'intégration des étrangers en

2)- Discours

de Jacques Chirac, président de la République, lundi 14 octobre 2002, Troyes (l'Aube).

Voir également Le Monde du 15 octobre 2002.
France et en Belgique.

Ainsi, évoquant la sécurité et le droit dans les cités, la politique de la ville et l'intégration lors d'un discours prononcé à Troyes en octobre $2002^{(2)}$, le président Chirac propose de donner une nouvelle vigueur au modèle d'intégration à la française. Ce faisant, et tout en précisant d'emblée que les 
réalités sont multiples derrière le mot intégration, ce dernier évoque la nécessite d'“accueillir dans de bonnes conditions les nouveaux arrivants" afin de les aider à "mieux s'insérer dans notre société", et surtout de développer "un véritable contrat d'intégration comprenant notamment la possibilité d'accéder à des formations et à un apprentissage rapide [de la langue française]". Plus précisément, ajoute quelques jours plus tard le cabinet du ministre des Affaires sociales François Fillon ${ }^{(3)}$, ce pacte engagerait le contractant à "suivre des cours de Français" et à apprendre "les droits et obligations civiques pendant une trentaine d'heures" afin d'expliquer aux migrants le fonctionnement des administrations et services publics français mais aussi de "leur rappeler le respect de l'ordre public, du code de la famille (interdiction de la polygamie ou des mariages forcés), la signification de la laïcité ou de la citoyenneté, le devoir de participer à la vie sociale et de ne pas s'enfermer dans le communautarisme..."

Derrière une définition très condensée de l'intégration, une définition qui exclut pêle-mêle les analphabètes, les incivils, les délinquants et tous ceux qui restent un peu trop chez eux ou qui ne maîtrisent pas les méandres de l'administration, Jacques Chirac s'engage sans aucune hésitation dans une logique de déplacement. Logique qui éclipse la question centrale de "ce à quoi nous voulons collectivement nous intégrer" et lui substitue la question secondaire de "la réussite ou de l'échec de l'intégration". Logique qui implique très clairement, pour être compréhensible, une définition négative de l'intégration, une définition qui ne fonctionne, n'existe que par son contraire. Ainsi, nous saurons juste qu'avec une formation civique et l'apprentissage de la langue française, la République rappellera à chacun qu'il n'a pas seulement des "droits mais aussi des devoirs, dont le plus essentiel est de respecter les lois de la République". République qui "ne saurait se dissoudre dans une mosä̈que de communautés". Oubliez l'opinion de Jacques Chirac et du gouvernement sur la société idéale à laquelle ils aspirent ! Oubliez donc la pensée présidentielle sur la société intégrée que Chirac administre! Ce n'est pas le but de son propos.

\section{Mauvais exemples}

Déplacer furtivement les enjeux du débat sur l'intégration vers la stigmatisation des "mauvais exemples" ou, plus sagement, vers les méthodes pour organiser cette dernière n'est pas un réflexe exclusivement de droite ou d'extrême droite. Ainsi, lors d'un discours au sujet de son projet de modification de la loi sur la nationalité à l'Assemblée nationale ${ }^{(4)}$, l'ancienne ministre de la Justice Elisabeth Guigou s'est efforcée de persuader les députés que pour faciliter l'intégration des jeunes étrangers nés en France de parents étrangers, il convenait de leur octroyer l'acquisition de plein droit de la nationalité française à dix-huit ans tout en laissant la place à la

4)- "Réforme de la justice, loi sur la nationalité", discours de madame Elisabeth Guigou, ministre de la Justice, Assemblée nationale, $1^{\text {er }}$ décembre 1997.

\section{3)- Le Monde,}

17 octobre 2002.

Voir également sur le site du Premier ministre

Raffarin : "Vers

une refondation du modèle français d'intégration", www.premierministre.gouv.fr 
ploit d'utiliser sept fois le concept d'intégration dans un document de six pages sans jamais offrir aux députés et aux lecteurs quelques précisions sur la société intégrée à laquelle ces jeunes étrangers devraient pouvoir s'intégrer en toute liberté. Sans consacrer une seule ligne à la société idéale à laquelle nous devrions aspirer. Sans jamais évoquer les qualités, les marques, la nature exacte du monde auquel nous appartenons, sans jamais véritablement définir de quoi il est question dans ce domaine, sans finalement apporter beaucoup plus de précisions que le projet intégrateur ultracondensé du président de la République. La question centrale, nous explique entre autres le Garde des Sceaux, est "celle de l'intégration [des jeunes étrangers] à la société française". C'est un débat "qui a trait à la solidarité, à la place de chacun dans la société et à la paix publique". Car l'acquisition de la nationalité est un "puissant

\begin{tabular}{|l|}
\hline $\begin{array}{l}\text { Quels sont les critères susceptibles } \\
\text { de définir les membres } \\
\text { de la "bonne"société intégrée? }\end{array}$ \\
\hline $\begin{array}{l}\text { Le caractère parfois farfelu de ces critères } \\
\text { promet de belles surprises. }\end{array}$
\end{tabular}
élément d'intégration à la société française", et sa logique ne peut être "celle de l'exclusion mais celle de l'intégration" ajoute la ministre maladroitement, contrainte, à défaut de pouvoir faire autrement, de définir l'intégration en l'opposant à son contraire.

Pour persuader les députés du bien-fondé de son projet de réforme de la loi sur la nationalité, Elizabeth Guigou s'engage directement, sans hésitation, dans une logique de déplacement. Logique qui éclipse, répétons-le encore, la question centrale de "ce à quoi nous voulons collectivement nous intégrer" et se rapporte directement à la question secondaire de "la réussite ou de l'échec de l'intégration", et à la gestion politique de cette dernière.

Déplacer discrètement les enjeux du débat sur l'intégration vers la stigmatisation des mauvais exemples d'intégration, ou plus sobrement vers les moyens pour administrer cette dernière, n'est pas un réflexe exclusive-

5)- Voir la circulaire du 14 juin 1999 concernant la modification du Code de la nationalité belge dans le Moniteur belge du 3 août 1999, à l'adresse suivante : www.moniteur.be ment français. Ainsi, jusqu'il y a peu, dans le cadre de certaines procédures relatives à l'acquisition de la nationalité belge ${ }^{(5)}$, le Code de la nationalité prévoyait notamment que le procureur du Roi puisse émettre un avis négatif sur l'acquisition de la nationalité, "lorsqu'il y a un empêchement résultant de faits personnels graves, ou s'il y a des raisons d'estimer que la volonté d'intégration du déclarant est insuffisante, ou encore lorsque les conditions de fond ne sont pas remplies". Concrètement, pour l'étranger désireux d'obtenir la nationalité belge, cela a signifié pendant des années qu'il fallait apporter la preuve de son intégration à la Belgique, et partant, qu'il fallait satisfaire, sur le terrain, à l'enquête menée par les autorités concernées. En pratique, celles-ci devaient alors considérer la participation à la vie sociale de l'étranger, ses loisirs (restaurants fréquentés, plats commandés, etc.), ses relations personnelles et professionnelles, sa famille, ses amis, ses lectures, ses choix en matière de programmes télévisés, ses activités le week-end, ses vêtements, etc., et statuer en conséquence. Autant de précautions laissées au bon vouloir des enquêteurs 
chargés de dire à leur autorité de tutelle s'il y avait eu ou non miracle de l'intégration" dans le chef de la personne concernée. Autant de précautions qui ont révélé de façon évidente l'absurdité de la logique du déplacement, et de l'acharnement qu'il implique à vouloir débattre de l'intégration sans s'être mis d'accord au préalable sur le monde dans lequel nous vivons et auquel nous aspirons.

\section{Débat déporté sur l'intégration}

Depuis le 7 juillet 1999, le gouvernement belge a déclaré que "la Belgique était une société ouverte et tolérante, dans laquelle le développement de la citoyenneté était à la base de l'intégration sociale [et que] l'acquisition de la nationalité belge [était] un facteur important pour réaliser une telle intégration". Ce faisant, il a modifié le Code de la nationalité et supprimé notamment l'obligation d'apporter la preuve "d'une volonté suffisante d'intégration" dans le cadre de la procédure d'acquisition de la nationalité belge $^{(6)}$. Ainsi, constatant l'absurdité et les dérives possibles d'une loi exigeant des étrangers la preuve de l'intégration, et faisant donc remarquer au passage les difficultés pour évaluer et définir cette dernière, le gouvernement, au nom de l'intégration en question, a supprimé cette disposition. S'il faut à l'évidence saluer une telle initiative, remarquons toutefois avec quelle persévérance le législateur belge s'acharne à éclipser la question centrale de "ce à quoi nous voulons collectivement nous intégrer" au profit de textes de loi et de circulaire évoquant encore et toujours, sans savoir officiellement de quoi l'on parle, l'intégration des étrangers ${ }^{(7)}$.

L'histoire de la phraséologie sur l'intégration n'a cessé de faire écho à cette logique du déplacement dans les débats, les discussions et les échanges de points de vue sur ces questions. Préférant batailler directement sur la réalité de l'intégration, la classe politique a forcément légitimé, au fil du temps, les opinions les plus diverses sur la réussite ou l'échec de cette dernière, ouvrant de cette manière des portes difficiles à refermer aujourd'hui. Ainsi, en France, profitant des débats interminables sur les critères d'intégration, le Front national a pu "légitimement" mettre la barre très haut en la matière, tout le monde ayant désormais sa petite idée sur la question!

"Pour s'assimiler, explique le programme du FN, il faut respecter et partager des valeurs spirituelles, morales, culturelles suffisamment fortes et entraînantes pour abandonner les siennes ${ }^{(8)}$ ". Ce faisant, le parti de JeanMarie Le Pen exige non pas une, mais deux preuves d'intégration : l'étranger ou l'immigré doit d'abord certifier qu'il n'est plus du tout sous l'influence des valeurs, des normes et des mœurs de son pays d'origine, il doit ensuite prouver qu'il marque et adopte sans hésitation les valeurs du pays d'accueil. Des valeurs qui trouvent leur source dans la France éternelle, "une nation venue du fond des âges [dont] la population est, pour l'essentiel, fixée depuis plus de deux millénaires". Des valeurs qui, d'après le
6)- Voir la circulaire du 25 avril 2000 concernant la loi du $1^{\text {er }}$ mars 2000 modifiant certaines

dispositions relatives à la nationalité belge dans le Moniteur belge du 6 mai 2000 à l'adresse suivante : www.moniteur.be ; Voir également Un combat pour les droits, rapport annuel 2000, Centre pour l'égalité des chances et la lutte contre le racisme, Bruxelles, p. 39 et suiv.

\section{7)- À la décharge}

du gouvernement belge, celui-ci pouvait difficilement faire l'économie d'une définition officielle de l'intégration. Depuis 1989 , le Commissariat royal

à la politique des immigrés (devenu le Centre

pour l'égalité des chances

et la lutte contre le racisme) a défini l'intégration à partir de trois niveaux : "Là où

l'ordre public, tel que la loi le définit, s'impose, c'est l'assimilation complète qui prévaut"; "Là où il s'agit de la diversité culturelle: respect sans équivoque en tant qu'enrichissement réciproque" et enfin plus globalement "promotion conséquente d'une insertion la plus poussée

conformément aux principes sociaux fondamentaux

soutenant la culture du pays d'accueil et tenant à la modernité, à l'émancipation et au pluralisme confirmé dans le sens d'un État occidental moderne". On y voit tout de suite plus clair... Voir (sous la dir. de Paula D'hondt et Bruno Vinikas), Lintégration: une politique de longue haleine, Commissariat royal à la politique des immigrés, novembre 1989 , vol. I, pp. 38-39.

8)- Extrait du programme du Front national disponible en format PDF à l'adresse suivante : www.frontnational.com/, p. 22. 
9)- Discours de Jean-Marie Le Pen à la dix-huitième fête des Bleu-Blanc-Rouge, septembre 1998

10)- Après avoir plaidé pour une séparation du Nord de l'Italie du reste de la Péninsule, le meneur d'extrême droite a obtenu dans le gouvernement de Berlusconi le portefeuille ministériel relatif aux réformes institutionnelles et à la décentralisation.

11)- Pour plus de détails, saisir la section Programme sur la page d'accueil du Front national belge à l'adresse suivante : www.frontnational.be. président du FN, ne se transmettent que par le sang et le corps : "De chair et de sang, avec nos cœurs, nos âmes, nos esprits, nous faisons partie par toutes nos fibres du corps mystique de la Patrie. C'est le sang de nos pères qui coule dans nos veines, le même qui a coulé pour la défense et la grandeur du pays. Ce sont les paysages, qu'ils ont préservés et embellis, qui sont le cadre de notre vie. C'est leur langue que nous parlons. Quand nous mangeons les produits de la terre, symbolisés par le pain et le vin, nous communions avec eux, dans le sentiment chaleureux de l'appartenance à la nation charnelle et spirituelle, et quand nos âmes quitteront l'ici-bas, c'est dans la terre maternelle que nous reposerons."(9) On imagine le travail abracadabrant des enquêteurs chargés de vérifier la double preuve de l'intégration si d'aventure, à l'image d'Umberto Bossi en Italie $^{(10)}$, un ou plusieurs leaders lepénistes obtenaient la tutelle des principaux ministères concernés par l'intégration des étrangers.

Pour devenir Belge, explique pour sa part le programme du Front national belge ${ }^{(11)}$, "le mode normal d'acquisition de la nationalité [...] doit demeurer la filiation : naît Belge tout enfant né de père ou de mère belge". Le droit du sang et rien d'autre! Il faut aussi une "demande expresse du candidat", il faut vérifier l'intégration de celui-ci ("entre autres : maîtrise d'une langue nationale, origine européenne"), réaliser une "enquête de moralité", demander "l'avis du conseil communal de la commune de résidence du candidat", exiger un "serment solennel de loyauté envers notre pays". La naturalisation, ajoute le programme, ne devient définitive qu'après une période probatoire de six ans. Enfin, il faut "appliquer la loi sur la déchéance de la nationalité" et surtout "rendre impossible la double nationalité".

Si nos responsables politiques s'accordent sur la hiérarchie tronquée du débat sur l'intégration, et surtout s'ils n'éprouvent aucune gêne à décrire les moyens d'une intégration réussie de façon aussi lapidaire et vide de sens, utilisant au passage des définitions négatives, il ne faut pas s'attendre à des différences trop marquées entre partis traditionnels et partis d'extrême droite dans ce domaine. Il ne faut pas non plus espérer des développements et des rebondissements extraordinaires en la matière. Mais si de surcroît ceux-ci s'accordent, toutes tendances confondues, à placer le respect des lois et de la République dans leur manifeste pour l'intégration, juste après les cours de langue (ne pouvant échapper ce faisant à l'irrésistible image de l'Arabe polygame qui frappe sa femme voilée et qui vole des mobylettes), il ne faut vraiment rien attendre de miraculeux sur ces questions dans les années à venir.

\section{Intégration et collectivité intégrée}

Avoir un emploi et payer ses impôts, respecter les lois de la République (ou de la Monarchie), avoir des enfants, aimer sa famille et sa culture, être en bonne santé et bien éduqué, parler français et avoir un logement salubre, suivre une formation et obtenir un diplôme, respecter le Code de la route 
constituent autant de critères susceptibles de définir les membres de la "bonne" société intégrée. Ils témoignent de la nécessité d'une mise au point dans l'ordre du discours et affichent l'urgence d'une table ronde, d'un dialogue authentique et sincère sur nos marqueurs d'appartenance. Labondance des indices et des traits identitaires qui nous caractérisent, ainsi que leurs nombreuses contradictions, imposent une mise au point qui ne pourra pas éternellement se dissimuler derrière une stigmatisation exclusive du jeune immigré délinquant, de la femme voilée et du patriarche polygame. "Qui sommes-nous ?" et "à quoi voulons-nous nous intégrer collectivement ?" sont véritablement les seules questions légitimes dans le champ de la réflexion sur l'intégration sociale.

Les critères susceptibles de définir les membres de la "bonne" société intégrée témoignent autant de la richesse des échanges possibles entre ceux qui choisiront de déterrer le fond du problème, que de l'aveuglement collectif des autres, qui s'obstineront à nier la complexité du social, complexité qui impose les limites d'une réflexion sur la nature exacte de notre communauté intégrée. Le caractère parfois farfelu de ce que pourra ou devra être un individu intégré promet d'ailleurs de belles surprises dans le chef des différentes sensibilités politiques et idéologiques, en France comme en Belgique. Quels que soient la nature et l'intérêt d'un débat sur ce que nous sommes et ce que nous voudrions être, la diversité et la variété des qualités identitaires affirmées ou revendiquées auront au moins le mérite de révéler le mauvais chemin suivi par ceux qui s'obstineront à vouloir batailler immédiatement sur "la réussite de l'intégration".

En définitive, ces qualités nous permettront d'identifier rigoureusement ceux qui, sans appel, cherchent à se définir négativement, en fondant leur identité sur la stigmatisation des autres. Ceux qui se protègent férocement d'un débat difficile, et perdu d'avance, sur leur définition trop étroite, et donc intenable, de la société et de la nation.

\begin{tabular}{|c|c|}
\hline \multirow[t]{5}{*}{ 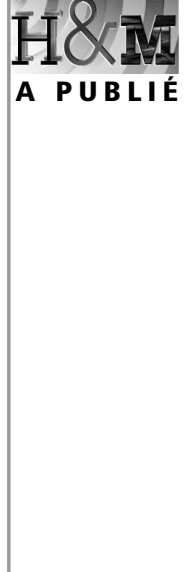 } & $\begin{array}{l}\text { Anne-Marie Gaillard, "Assimilation, insertion, intégration, adaptation : } \\
\text { un état des connaissances" } \\
\text { Hors-dossier, } n^{\circ} 1209 \text {, septembre-octobre } 1997\end{array}$ \\
\hline & $\begin{array}{l}\text { Pierre Yves Cusset, "Le modèle français d'intégration est-il en crise ?" } \\
\text { Laure-Leyla Chebbah, "La politique française d'intégration, } \\
\text { entre spécifique et droit commun" } \\
\text { Dossier Intégration et politique de la ville, n 1203, novembre } 1996\end{array}$ \\
\hline & $\begin{array}{l}\text { Marco Martiniello, "Philosophies de l'intégration en Belgique" } \\
\text { — Dossier Détours européens, n 1193, décembre } 1995\end{array}$ \\
\hline & $\begin{array}{l}\text { Abdelmalek Sayad, "Qu'est-ce que l'intégration ?" } \\
\text { Rochdy Alili, "Qui s'intègre à quoi ? Qui intègre qui ?" } \\
\text { Dossier Pour une éthique de l'intégration, n 1182, décembre } 1994\end{array}$ \\
\hline & $\begin{array}{l}\text { Françoise Gaspard, "Assimilation, insertion, intégration : les mots pour 'devenir français'" } \\
\text { Simone Bonnafous, "Le terme 'intégration' dans le journal Le Monde : sens et non-sens" } \\
\text { - Dossier Le poids des mots, n } 1154, \text { mai } 1992\end{array}$ \\
\hline
\end{tabular}

